\title{
346.
}

\section{NOTE ON AN EXPRESSION FOR THE RESULTANT OF TWO BINARY CUBICS.}

[From the Quarterly Journal of Pure and Applied Mathematics, vol. vi. (1864), pp. $380-382$.

Mr Warren, in his paper "Illustrations of the Theory of Critical Functions," Quarterly Mathematical Journal, t. vi. pp. 231-237, (1864), has given for the Resultant of two binary cubic functiols, an expression which is in effect as follows; viz. considering the cubic

its Hessian

$$
\left(a, b, c, d \gamma(x, y)^{3},\right.
$$

$$
\left(\mathrm{a}, \mathrm{b}, \mathrm{c} \gamma(x, y)^{2},=\left(a c-b^{2}, a d-b c, b d-c^{2} \gamma(x, y)^{2},\right.\right.
$$

and the cubicovariant

$$
\left(A, B, C, D \gamma(x, y)^{3},=\left\{\begin{array}{r}
a^{2} d-3 a b c+2 b^{3} \\
3 a b d-6 a c^{2}+3 b^{2} c \\
-3 a c d+6 b^{2} d-3 b c^{2} \\
-a d^{2}+3 b c d-2 c^{3}
\end{array}\right\}(x, y)^{3}\right.
$$

and in like manner the cubic

its Hessian

$$
\left(a^{\prime}, b^{\prime}, c^{\prime}, d^{\prime} \gamma(x, y)^{3}\right. \text {, }
$$

$$
\left.\left(\mathrm{a}^{\prime}, \mathrm{b}^{\prime}, \mathrm{c}^{\prime}\right\} x, y\right)^{2},
$$

and the cubicovariant

and writing

$$
\left(A^{\prime}, B^{\prime}, C^{\prime}, D^{\prime} \chi x, y\right)^{3} \text {; }
$$

$$
\begin{aligned}
& \mathfrak{A}=a d^{\prime}-3 b c^{\prime}-3 b^{\prime} c-a^{\prime} d, \\
& \mathfrak{B}=a c^{\prime}+\mathrm{a}^{\prime} \mathrm{c}-2 \mathrm{bb}^{\prime}, \\
& \mathfrak{E}=A D^{\prime}-3 B C^{\prime}+3 B^{\prime} C-A^{\prime} D,
\end{aligned}
$$

C. V. 
then the Resultant is

$$
=-2 \mathfrak{A}^{3}+27 \mathfrak{A} \mathfrak{B}+27 \mathfrak{c},
$$

that is, the Resultant is

$$
\begin{aligned}
= & -2\left(a d^{\prime}-a^{\prime} d-3 b c^{\prime}+3 b^{\prime} c\right)^{3} \\
+ & 27\left(a d^{\prime}-a^{\prime} d-3 b c^{\prime}+3 b^{\prime} c\right) \times \\
& \left\{\left(a c-b^{2}\right)\left(b^{\prime} c^{\prime}-d^{\prime 2}\right)-\frac{1}{2}(a d-b c)\left(a^{\prime} d^{\prime}-b^{\prime} c^{\prime}\right)+\left(b d-c^{2}\right)\left(a^{\prime} c^{\prime}-b^{\prime 2}\right)\right\} \\
+27 & \left(a^{2} d-3 a b c+2 b^{3}\right)\left(-a^{\prime} d^{\prime 2}+3 b^{\prime} c^{\prime} d^{\prime}-2 c^{\prime 3}\right) \\
& -3\left(a b d-2 a c^{2}+b^{2} c\right)\left(-a^{\prime} c^{\prime} d^{\prime}+2 b^{\prime 2} d^{\prime}-b^{\prime} c^{\prime 2}\right) \\
& +3\left(-a c d+2 b^{2} d-b c^{2}\right)\left(a^{\prime} b^{\prime} d^{\prime}-2 a^{\prime} c^{\prime 2}+b^{\prime 2} c\right) \\
& \left.-\left(-a d^{2}+3 b c d-2 c^{3}\right)\left(a^{\prime 2} d^{\prime}-3 a^{\prime} b^{\prime} c^{\prime}+2 b^{\prime 3}\right)\right\} .
\end{aligned}
$$

In particular assume

so that

$$
\left(a^{\prime}, b^{\prime}, c^{\prime}, d^{\prime} \chi x, y\right)^{3}=x^{3}+y^{3},
$$

and thus

$$
\begin{aligned}
\left(\mathrm{a}^{\prime}, \mathrm{b}^{\prime}, \mathrm{c}^{\prime} \gamma(x, y)^{2}\right. & =x y, \\
\left(A^{\prime}, B^{\prime}, C^{\prime}, D^{\prime} \gamma(x, y)^{3}\right. & =x^{3}-y^{3},
\end{aligned}
$$

$$
\begin{aligned}
& \begin{aligned}
a^{\prime}=d^{\prime}=1, & b^{\prime}=c^{\prime}=0, \\
a^{\prime}=c^{\prime}=0, & b^{\prime}=\frac{1}{2}, \\
A^{\prime}=-D^{\prime}=1, & B^{\prime}=C^{\prime}=0 .
\end{aligned} \\
& \mathfrak{A}=a-d, \\
& \mathfrak{B}=-\mathrm{b}=b c-a d, \\
& \mathfrak{C}=A+D=a^{2} d-a d^{2}-3 a b c+3 b c d+2 b^{3}-2 c^{3},
\end{aligned}
$$

or, putting for shortness,

$$
a-d=\theta, \text { and therefore } a=\bar{d}+\theta,
$$

we have

$$
\begin{aligned}
& \mathfrak{U}=\theta \\
& \mathfrak{B}=b c-d \theta-d^{2}, \\
& \mathfrak{E}=2\left(b^{3}-c^{3}\right)-3 b c \theta+d^{2} \theta+d \theta^{2},
\end{aligned}
$$

and Resultant is

$$
\begin{aligned}
& -2 \theta^{3} \\
& +27 \theta\left(b c-d^{2}-d \theta\right) \\
& +27\left\{2\left(b^{3}-c^{3}\right)-3 b c \theta+d^{2} \theta+d \theta^{2}\right\}
\end{aligned}
$$

which is

$$
=-2 \theta^{3}+54 b^{3}-54 c^{3}-54 b c \theta,
$$

or rejecting the factor -2 , it is

$$
=\theta^{3}-27 b^{3}+27 c^{3}+27 c b \theta \text {. }
$$


But the two equations are

$$
\begin{aligned}
\left(a, b, c, d \gamma(x, y)^{3}\right. & =0, \\
x^{3}+y^{3} & =0,
\end{aligned}
$$

the last of which gives $y=-x, y=-\omega x, y=-\omega^{2} x$, if $\omega$ be an imaginary cube root of unity, and hence the Resultant is

$$
=(a-3 b+3 c-d)\left(a-3 b \omega+3 c \omega^{2}-d\right)\left(a-3 b \omega^{2}+3 c \omega-d\right),
$$

which is

$$
=(\theta-3 b+3 c)\left(\theta-3 b \omega+3 c \omega^{2}\right)\left(\theta-3 b \omega^{2}+3 c \omega\right),
$$

or finally is

$$
=\theta^{3}-27 b^{3}+27 c^{3}+27 b c \theta,
$$

and the formula is thus verified.

If the two cubies are taken to be

$$
\begin{aligned}
& \left(a, b, c, d \gamma(x, y)^{3}=0,\right. \\
& \left(b, c, d, e \chi(x, y)^{3}=0,\right.
\end{aligned}
$$

then the formula gives for the Discriminant of the quartic function $\left(a, b, c, d, e \gamma(x, y)^{4}\right.$ a new expression, which however does not appear to be an elegant one. 